\title{
Impacto nutricio del consumo de una leche entera adicionada con vitaminas y minerales en niños
}

Irene Maulen-Radovan, M.C., ${ }^{(1)}$ Sandra Villagómez, M.C., ${ }^{(2)}$ Esther Soler, M.C., ${ }^{(2)}$

Rolando Villicaña, M.C., ${ }^{(3)}$ Lizbeth Hernández-Ronquillo, M.C. .(1) Jorge L. Rosado, M. en C., D r. en C. ${ }^{(5)}$

\section{Maulen-Radovan I, Villagómez S, Soler E, Villicaña R, Hernández-Ronquillo L, Rosado JL. Impacto nutricio del consumo de una leche entera adicionada con vitaminas y minerales en niños. Salud Publica Mex 1999;41:389-396.}

\section{Resumen}

Objetivo. Determinar el impacto nutricio del consumo de leche entera fortificada con vitaminas y minerales en niños. Material y métodos. Se hizo un estudio prospectivo, longitudinal, en 227 niños de entre 8 a 60 meses de edad. Se ofreció a los menores $500 \mathrm{ml}$ diarios de leche entera fortificada por 90 días.Se registró ingestión, aceptación, peso, talla, hemoglobina $(\mathrm{Hb})$, hier ro (Fe), vitamina $\mathrm{B}_{12}$ y folatos séricos. El análisis estadístico se realizó con medidas de tendencia central y dispersión en variables dimensionales utilizando pruebat de Student para comparación de promedios y $\chi^{2}$ para variables nominales. Resultados. Al inicio de la suplementación 45 niños estaban desnutridos, y 36 , anémicos. Al final de la misma estas cifras disminuyeron: 35 desnutridos $(p<0.21)$ y 18 niños anémicos $(p<0.01)$.Al inicio nueve niños tenían desnutrición severa y, al finalizar, sólo eran cinco los que la padecían. La comparación ingreso-egreso en los datos antropométricos fue como sigue (media \pm desviación estándar): $Z$ peso/talla, $-0.35 \pm 0.88$ vs. $-0.14 \pm 0.9(p=0.01) ; \mathrm{Hb}$ en $\mathrm{g} / \mathrm{dl}, 11 \pm 1.3$ vs. $11.9 \pm 1.9$ $(p<0.001) ;$ Fe en $\mathrm{mg} / \mathrm{dl}, 108 \pm 44$ vs. $115 \pm 31(p=0.06)$; vitamina $\mathrm{B}_{1}$ en $\mathrm{pg} / \mathrm{ml}, 649 \pm 494$ vs. $1053 \pm 854$ ( $p<0.001$ ). El apego y la aceptación fueron de 100 y $85 \%$, respectivamente. Conclusiones El consumo de leche entera fortificada durante 90 días mejora significativamente el estado nutricio de los niños, reduce significativamente el número de niños con anemia e incrementa los niveles plasmáticos de $\mathrm{Hb}$, Vitamina $\mathrm{B}_{12} \mathrm{y}$ folatos.

Palabras clave:leche; alimentos fortificados; micronutrientes; desnutrición; niños; México

\section{Maulen-Radovan I, Villagómez S,}

Soler E, Villicaña R,

Hernández-Ronquillo L, Rosado JL.

Nutritional impact of a full strenght milk with added

vitamins and minerals in children.

Salud Publica Mex 1999;41:389-396.

\begin{abstract}
A bstract
Objective. To evaluate the nutritional impact of the ingestion of a fortified whole milk in children. Material and methods Prospective, longitudinal assay in 227 children aged 8-60 months. Intervention: D aily consumption of 500 $\mathrm{ml}$ of fortified milk during 90 days. We registered milk acceptance and assessed weight, height; hemoglobin, serum iron, vitamin $\mathrm{B}_{12}$, and folic acid, at the beginning and the end of the study. Statistical evaluation were done with central and dispersion indices in the dimensional variables, using Student's test and $\chi^{2}$ test for compare nominal variables at initial and the end of the study. Results. At admission, 45 children were malnourished and 36 were anemic. At the end of the supplementation period there was a reduction to 35 malnourished $(p<0.21)$ and 18 anemic $(p<0.01)$. Anthropometric weight/height score in $Z$ at the beginning and end of the study $(x \pm S . D)$ were $-0.35 \pm 0.88$ vs $0.14 \pm 9(p<0.01) ; \mathrm{Hb} g / d l: 11 \pm 1.3$ vs $11.9 \pm 1.9(p<0.001)$, Iron mg/dl: $108 \pm 44$ vs $115 \pm 31(p=0.06)$ and vitamin $B_{1}$ $\mathrm{pg} / \mathrm{ml}: 649 \pm 494$ to $1053 \pm 854(p<0.001)$. The milk was welf tolerated and widely accepted. Conclusions. The consumption of a fortified whole milk during 90 days improved significantly the nutritional status of the children, the weight for height $Z$ score, the plasma level of vitamin $B_{12}$ and $\mathrm{Hb}$, and decreased the number of anemic and malnourished children.
\end{abstract}

Key words: $N$ utritional supplement; vitamins; micronutrients; malnourished; children; Mexico

(1) División de Investigación, Instituto $\mathrm{N}$ acional de Pediatría (IN P), México.

(2) Unidad Metabólica, IN P, México.

(3) Laboratorio de Investigación, IN P, México.

(4) Residente de Pediatría, IN P, México.

(5) Departamento de Fisiología de la N utrición, Instituto N acional de la N utrición Salvador Zubirán, México.

Fecha de recibido: 16 de febrero de 1999 - Fecha de aprobado: 14 de junio de 1999

Solicitud de sobretiros: D ra. Irene Maulen-Radovan. Insurgentes Sur 3700-C, Insurgentes Cuicuilco, 04720 México, D.F., México. Correo electrónico: maulen@ cenids.ssa.gob.mx 
$\mathrm{L}^{2}$ as deficiencias vitamínicas o de micronutrientes tienen un impacto negativo en la salud infantil. ${ }^{1-5}$ Varios informes muestran la asociación de deficiencias vitamínicas y de micronutrimentos con una menor respuesta inmune, con el aumento de la frecuencia de infecciones respiratorias y digestivas, y con alteraciones en las habilidades cognitivas. ${ }^{6-9}$

Existe información parcial de la magnitud de deficiencias de micronutrimentos y vitaminas en niños mexicanos. Aquella disponible demuestra que existe deficiencia de hierro, retinol, vitamina E, riboflavina y vitamina $\mathrm{B}_{12}{ }^{10-13}$ La segunda Encuesta Nacional de Alimentación en el Medio Rural de 1989, notificó que existe un consumo insuficiente de niacina, riboflavina, retinol y ácido ascórbico. ${ }^{14}$

La deficiencia de hierro tiene una elevada prevalencia en el ámbito mundial y también en nuestro país. Se conoce que los grupos de más riesgo son los niños menores de dos años y las mujeres embarazadas. Información reciente señala que en México existe una prevalencia de anemia por deficiencia de hierro de $50 \%$ entre los niños de 6 a 36 meses de edad, la cual puede corregirse al dar suplementos con hierro. ${ }^{15}$ Este tipo de anemia en lactantes y prescolares está asociada con una disminuida resistencia a infecciones y un retardo en el crecimiento y en el desarrollo cognoscitivo; estos efectos son irreversibles aun cuando se corrija la deficiencia. $^{16-19}$

La deficiencia de micronutrimentos ha sido reconocida en diversos países, especialmente en aquellos en vías de desarrollo, y se sabe que tiene graves repercusiones sobre la salud. Posterior a la Conferencia sobre Nutrición de la Organización para la Agricultura y la Alimentación/Organización Mundial de la Salud (FAO/OMS), en 1992, en Roma, se ha recomendado e implementado en diversas regiones del mundo un gran número de iniciativas de adición de vitaminas y minerales a alimentos de amplio consumo, para evitar y corregir las deficiencias nutricias de mayor prevalencia, como las de vitamina A, hierro y yodo. ${ }^{20}$

La adición de vitaminas y minerales a los alimentos comunes es la mejor estrategia para corregir el nivel de micronutrimentos de grandes sectores de la población, pues no se requiere de modificar la dieta habitual ni de insistir en el cumplimiento individual de un programa nuevo; es un método que se puede seguir por periodos prolongados. Por lo anterior, la adición de vitaminas y minerales a los alimentos puede ser puesta en práctica de forma más económica para superar la desnutrición por deficiencia de minerales y vitaminas.

Algunos autores recomiendan la suplementación con múltiples micronutrimentos, ya que en la mayoría de las ocasiones los niños presentan deficiencia de más de dos micronutrimentos y porque se logra un mayor impacto nutricio..$^{10} \mathrm{La}$ OMS promueve la fortificación con micronutrimentos en alimentos de consumo masivo, de bajo costo y disponibles en el mercado para que lleguen de forma suficiente a los grupos de población en riesgo. Otra estrategia recomendable es la entrega de suplementos con hierro a mujeres embarazadas y a niños menores de dos años. ${ }^{21}$

En México existen diversas estrategias del sector salud para reducir la malnutrición y las deficiencias de micronutrimentos en niños menores de cinco años. El Programa de Educación, Salud y Alimentación (PROGRESA) emplea un suplemento lácteo adicionado con las vitaminas A, E, C, $\mathrm{B}_{2^{\prime}} \mathrm{B}_{12^{\prime}}$ ácido fólico y los minerales hierro, yodo y zinc para niños menores de cinco años; también utiliza otro para mujeres embarazadas y en lactancia, con similar composición pero sin vitamina A ni vitamina $B_{2}$. La distribución a familias de extrema pobreza es gratuita. ${ }^{22}$ Otras estrategias en México son la fortificación con hierro de las harinas de trigo y maíz y el programa de desayunos escolares.

Otras vertientes de solución a las deficiencias de micronutrimentos y vitaminas son los diversos esfuerzos de la industria alimentaria, la que participa mediante la fortificación de sus productos; de este modo, desde hace más de dos décadas se han fortificado alimentos industrializados de venta al público. Este estudio evalúa el impacto del consumo de una leche entera adicionada con múltiples micronutrimentos, de venta al público, lista para su uso como suplemento nutricio entre los niños.

\section{Material y métodos}

Se hizo un estudio prospectivo, longitudinal, aprobado por las Comisiones de Investigación y Etica del Instituto Nacional de Pediatría, entre 227 niños, residentes de la colonia Pedregal de las Águilas, Delegación Tlalpan, en el sureste de la ciudad de México. Se invitó a participar a los menores que asistían a las escuelas y jardines de niños, con una edad de entre 8 meses y 4 años, independientemente del género, y que no estuvieran lactando al seno materno; también se cuidó que no presentaran malformaciones congénitas, enfermedades crónicas (como reflujo gastroesofágico, mala absorción intestinal, intolerancia a la lactosa o alergia) y sobrepeso ( $Z$ peso/talla $>2$ ). En todos los casos se solicitó el consentimiento informado de los padres. Los niños en estudio fueron citados en el Centro de Salud Las Águilas, que es un centro de atención de primer nivel, para reconocer su elegibilidad por criterios de 
inclusión, exclusión y también durante el seguimiento. La investigación se realizó de marzo de 1998 a julio de 1998.

El estudio socioeconómico de las familias mostró viviendas con paredes de ladrillo o concreto en $90.4 \%$ de los casos, agua potable en $83.1 \%$ y drenaje en $68.9 \%$. En la investigación participaron 108 hombres y 119 mujeres. A los niños elegibles se les realizó un examen coproparasitoscópico, en serie de tres, con el método de Faust. A los niños que estaban parasitados se les administró tratamiento específico y, al final del mismo, se les realizó un coproparasitoscópico de control; cuando se constató que ya no tenían parásitos se les incluyó en el estudio. Se determinó el peso con una báscula electrónica con sensibilidad de 10 gramos, y la talla, en los menores de dos años, con un infantómetro; a los mayores de esta edad se les midió la estatura de pie. El personal médico y de enfermería fue previamente capacitado y estandarizado para la realización de estas mediciones.

Se determinó la concentración de hemoglobina (método colorimétrico semiautomatizado ${ }^{23}$ en equipo Sysmex F-800), hierro sérico, captación total y saturación de hierro (espectrofotometría) ${ }^{24}$ por el método de Beale y Loria; la vitamina $\mathrm{B}_{12}$ y los folatos (radioinmunoensayo ${ }^{25}$ se midieron con el Kit Simul TRAC-SNB, ICN Pharmaceuticals. Se tomaron las muestras sanguíneas con jeringa estéril a las ocho de la mañana, después de un ayuno de seis horas. Para la determinación de vitamina $B_{12}$ y folatos, la muestra fue colocada en un microtubo heparinizado con tapa de rosca, centrifugada a $2500 \mathrm{rpm}$, durante 15 minutos, a fin de separar el plasma, el cual se colocó en un microtubo con tapa y se guardó a $-20^{\circ} \mathrm{C}$ hasta el momento de procesarse (menos de siete días).

Los familiares de los niños fueron capacitados para el llenado de una hoja de recordatorio de 24 horas, donde se registró diariamente el volumen de leche consumido en 24 horas, la presencia de efectos adversos (cambio en las características de las evacuaciones, flatulencia o distensión abdominal) y la aceptación. Durante 90 días, se proporcionó la cantidad de $1000 \mathrm{ml}$ diarios de leche entera ultrapasteurizada y fortificada con micronutrimentos, con la composición que se muestra en el cuadro I; se pidió el consumo de al menos $500 \mathrm{ml}$ diarios durante 90 días consecutivos. Dos trabajadores sociales y dos enfermeras realizaron visitas domiciliarias a los niños dos veces a la semana para corroborar el consumo de la leche y el llenado de la hoja de recordatorio. Se citó a los niños en el centro de salud cada 15 días para la medición de peso y talla, la revisión de la hoja de recordatorio, la revisión médica y la entrega de la dotación de leche.

\section{Cuadro I \\ Composición de LA LeCHE ADICIONADA CON VitAminaS Y Minerales. Ciudad de MÉxico, 1998}

Nutrimentos Cantidad*

\begin{tabular}{ll} 
Proteínas & $15 \mathrm{~g}$ \\
\hline Carbohidratos & $40 \mathrm{~g}$ \\
\hline Grasa & $15 \mathrm{~g}$ \\
\hline Energía & $355 \mathrm{Kcal}$ \\
\hline Sodio & $200 \mathrm{mg}$ \\
\hline Hierro & $1.5 \mathrm{mg}$ \\
\hline Vitamina A & $7500 \mathrm{UI}$ \\
\hline Vitamina D & $600 \mathrm{UI}$ \\
\hline Vitamina C & $105 \mathrm{mg}$ \\
\hline Vitamina $\mathrm{B}_{6}$ & $3 \mathrm{mg}$ \\
\hline Vitamina $\mathrm{B}_{12}$ & $3 \mathrm{mg}$ \\
\hline Niacina & $25.5 \mathrm{mg}$ \\
\hline Ácido fólico & $2 \mathrm{mg}$ \\
\hline Biotina & $0.05 \mathrm{mg}$ \\
\hline Zinc & $22.5 \mathrm{mg}$ \\
\hline Yodo & $0.22 \mathrm{mg}$
\end{tabular}

* Nutrimentos por $500 \mathrm{ml}$

Se consideraron como variables de respuesta la modificación ingreso-egreso de los índices antropométricos ( $\mathrm{Z}$ peso/talla, $\mathrm{Z}$ talla/edad, $\mathrm{Z}$ peso/edad), de las concentraciones séricas de hierro, ácido fólico, vitamina $\mathrm{B}_{12} \mathrm{y}$ hemoglobina, así como de la aceptación y del apego al consumo de la leche fortificada. Se consideró que había desnutrición cuando el índice peso para la talla $(\mathrm{Z}$ p/t) era $<-1 \mathrm{Z}$; desnutrición leve, cuando $\mathrm{Z}$ $\mathrm{p} / \mathrm{t}$ era de entre -1 y -1.99 , y desnutrición moderada/ severa, cuando el índice $\mathrm{p} / \mathrm{t}$ era $<-2 \mathrm{Z}$. Se determinó desmedro cuando el índice talla / edad fue $<-1 \mathrm{Z}$, y emaciación, cuando se presentaban niños con índice peso / edad $<-1$ Z. Se definió el apego como el porcentaje de mililitros de leche consumidos/los mililitros de leche recomendados ( $500 \mathrm{ml}$ al día durante 90 días), y la aceptación, como el porcentaje de días de consumo de leche fortificada del total recomendado (120 días). Se consideró que había anemia cuando la hemoglobina plasmática era $<10 \mathrm{~g} / \mathrm{dl}$; deficiencia de hierro, cuando las concentraciones plasmáticas de hierro sérico eran menores a $80 \mathrm{mg} / \mathrm{dl}$; deficiencia de vitamina $\mathrm{B}_{12^{\prime}}$ cuando las concentraciones séricas eran $<200 \mathrm{pg} / \mathrm{ml}$, y deficiencia de ácido fólico, cuando los niveles séricos eran $<3 \mathrm{ng} /$ dl. Asimismo, se definió finalización del seguimiento (egreso) al completar 90 días desde la inclusión en el estudio. 
El cálculo de los índices antropométricos se efectuó con el programa Epi Info 6 de la OMS y de los Centros para la Prevención y el Control de las Enfermedades de Atlanta. El análisis estadístico se realizó en computadora personal IBM, compatible con el programa Epi Info de la OMS. Se efectuó un análisis descriptivo mediante media y desviación estándar (DE), para las variables continuas, y número de pacientes y porcentaje, para las variables categóricas. La comparación de variables de respuesta inicio-final se efectuó mediante la prueba $t$ de Student, y para las variables independientes se utilizó la U de Mann Whitney.

\section{Resultados}

Las características de los niños al inicio del estudio se muestran en los cuadros II y III. Cincuenta y tres niños elegibles estaban parasitados (32 niños con Giardia lamblia, 13 con Ascaris lumbricoides, cinco con Himenolepis, uno con Entamoeba hystolitica, otro con Trichuris trichiura y uno más con Estrongiloides estercoralis) y recibieron antiparasitarios antes de ser incluidos en el estudio.

La aceptación de la leche fue adecuada, y cuando se le comparó de acuerdo con el estado nutricio de los niños, resultó similar: de $87.5 \pm 19.2 \%$ en el grupo de niños desnutridos, y de $87.6 \pm 19 \%$ en el grupo con estado nutricio adecuado. El apego al consumo fue de $100 \%$ tanto en el grupo de niños desnutridos, como en los de estado nutricio adecuado.

Los índices antropométricos y los indicadores bioquímicos del estado de nutrición al ingreso y cuando se concluyó la suplementación se muestran en el cuadro IV. El índice $Z$ peso / talla se modificó de $-0.35 \pm .88$ a $0.14 \pm .90$, es decir, mejoró significativamente; el índice $\mathrm{Z}$ talla/edad resultó similar, pues sólo varió de $-0.81 \pm$ 1.11 a $-0.92 \pm 1.07$, y el índice $Z$ peso/edad tampoco fue diferente ( $-0.85 \pm .98$ a $-0.72 \pm 1)$. La hemoglobina, el número de niños anémicos, el número de niños con deficiencia de hierro y las concentraciones plasmáticas de ácido fólico y vitamina $B_{12}$ mejoraron significativamente al egreso. El número de niños desnutridos no presentó diferencias estadísticas significativas al finalizar la suplementación en comparación con el ingreso; sin embargo, cuando se observó el movimiento del estado nutricio en general, es decir, aquellos con desnutrición severa a leve o aquellos que pasaron de una desnutrición leve a un estado nutricio normal (cuadro $\mathrm{V})$, las diferencias fueron estadísticamente significativas.

\section{Cuadro II \\ Características de la población al inicio del estudio.* Ciudad de México, 1998}

\begin{tabular}{|c|c|c|c|c|}
\hline Variable & $\begin{array}{c}\text { Hombres } \\
n=108\end{array}$ & $\begin{array}{c}\text { Mujeres } \\
n=119\end{array}$ & $p$ & $\begin{array}{l}\text { Todos } \\
n=227\end{array}$ \\
\hline Edad (meses) & $31.1 \pm 14.9$ & $31 \pm 14$ & NS & $31 \pm 14.4$ \\
\hline $\mathrm{N}$ iños de 8-24 meses (41\%) & $15.02 \pm 4.8$ & $16 \pm 4.9$ & NS & $15.5 \pm 4.9$ \\
\hline $25-41$ meses $(31 \%)$ & $31.8 \pm 4.6$ & $32.9 \pm 3.8$ & NS & $32.47 \pm 4.2$ \\
\hline $42-60$ meses $(28 \%)$ & $48.36 \pm 5.1$ & $49.41 \pm 5.1$ & NS & $48.85 \pm 5.1$ \\
\hline Peso (kg) & $12.1 \pm 2.9$ & $12 \pm 2.8$ & NS & $12.3 \pm 3$ \\
\hline Talla (cm) & $88 \pm 11.3$ & $86.6 \pm 10.9$ & NS & $86.4 \pm 11.1$ \\
\hline Z peso/talla & $-0.45 \pm 0.82$ & $-.27 \pm .93$ & NS & $-.35 \pm 0.88$ \\
\hline $\mathrm{N}$ iños con peso/talla $<-1 Z(\%)$ & $22(20.3)$ & $23(19.3)$ & NS & $45(19.8)$ \\
\hline $\mathrm{N}$ iños con peso/talla $<-2 \mathrm{Z}(\%)$ & $4(3.7)$ & $5(4.2)$ & NS & $9(3.9)$ \\
\hline Z Talla/edad & $-.78 \pm 1.15$ & $-.87 \pm 1.08$ & NS & $-.81 \pm 1.11$ \\
\hline $\mathrm{N}$ iños con talla/edad <-1Z (\%) & $52(43.6)$ & $58(48.7)$ & NS & $110(48.4)$ \\
\hline $\mathrm{N}$ iños con talla/edad <-2Z (\%) & $15(12.3)$ & $13(10.9)$ & NS & $28(12.3)$ \\
\hline Z Peso/edad & $-.85 \pm .98$ & $-.80 \pm 1$ & NS & $-0.85 \pm .98$ \\
\hline $\mathrm{N}$ iños con peso/edad <-1Z (\%) & $48(44.4)$ & $54(45.3)$ & NS & $102(44.9)$ \\
\hline $\mathrm{N}$ iños con peso/edad <-2Z (\%) & $15(12.3)$ & $13(10.9)$ & NS & $28(12.3)$ \\
\hline
\end{tabular}




\section{Características de la población al inicio del estudio.* Ciudad de México, 1998}

\begin{tabular}{|c|c|c|c|c|}
\hline Variable & $\begin{array}{c}\text { Hombres } \\
n=108\end{array}$ & $\begin{array}{l}\text { Mujeres } \\
n=119\end{array}$ & p & $\begin{array}{c}\text { Todos } \\
n=227\end{array}$ \\
\hline Hemoglobina, g/dl & $11.1 \pm 1.4$ & $11 \pm 1.3$ & NS & $11 \pm 1.3$ \\
\hline $\mathrm{N}$ iños anémicos (\%) & $14(12.9)$ & $22(18.4)$ & NS & $36(15.8)$ \\
\hline Hierro sérico, $\mu \mathrm{g} / \mathrm{dl}$ & $107.8 \pm 43.5$ & $108.3 \pm 44.6$ & NS & $108 \pm 44$ \\
\hline $\mathrm{N}$ iños deficientes en Fe (\%) & $26(24)$ & $32(26.8)$ & NS & $58(25.5)$ \\
\hline Saturación hierro, \% & $31.9 \pm 14.3$ & $31.1 \pm 16.2$ & NS & $31.5 \pm 15.3$ \\
\hline Captación total de hierro, $\mu \mathrm{g} / \mathrm{dl}$ & $348 \pm 105$ & $353 \pm 107$ & NS & $351 \pm 105$ \\
\hline Acido fólico, $\mu \mathrm{g} / \mathrm{ml}$ & $12.9 \pm 8.5$ & $12.6 \pm 7$ & NS & $12.8 \pm 8$ \\
\hline $\mathrm{N}$ iños deficientes en folatos (\%) & $4(3.7)$ & $2(1.6)$ & NS & $6(2.6)$ \\
\hline Vitamina $\mathrm{B}_{12}, \mathrm{pg} / \mathrm{ml}$ & $632.6 \pm 448.8$ & $663.9 \pm 533.4$ & NS & $648.9 \pm 494$ \\
\hline $\mathrm{N}$ iños deficientes en vitaminas $\mathrm{B}_{12}(\%)$ & $14(12.9)$ & $22(18.5)$ & NS & $36(15.8)$ \\
\hline N iños parasitados (\%) & $26(24)$ & $27(22.6)$ & NS & $53(23.3)$ \\
\hline
\end{tabular}

*Datos notificados como $\bar{X} \pm D E$, a menos que se especifique otra medición $N$ S: sin diferencia estadísticamente significativa

\section{Cuadro IV}

VARIACIÓN DE ÍNDICES ANTROPOMÉTRICOS E INDICADORES BIOQUíMICOS DEL ESTADO NUTRICIO

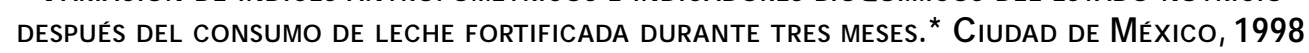

\begin{tabular}{|c|c|c|c|c|}
\hline Variable & $\begin{array}{l}\text { Inicial } \\
n=227\end{array}$ & $\begin{array}{c}\text { Final } \\
n=227\end{array}$ & $\begin{array}{l}\text { Diferencia } \\
\text { inicial- final }\end{array}$ & $p$ \\
\hline Z peso/talla & $-0.35 \pm .88$ & $-0.14 \pm .90$ & $0.24 \pm .55$ & 0.012 \\
\hline $\mathrm{N}$ iños con peso/talla <-1Z (\%) & $45(19.8)$ & $35(15.4)$ & $10(4)$ & 0.21 \\
\hline $\mathrm{N}$ iños con peso/talla<-2Z (\%) & $9(3.9)$ & $5(2.2)$ & $4(.7)$ & 0.27 \\
\hline Z talla/edad & $-0.81 \pm 1.11$ & $-0.92 \pm 1.07$ & $0.16 \pm .37$ & 0.28 \\
\hline $\mathrm{N}$ iños $<-1 Z$ talla /edad (\%) & $110(48)$ & $110(48)$ & $0(0)$ & 1 \\
\hline Z peso/edad & $-0.85 \pm .98$ & $-0.72 \pm 1$ & $0.11 \pm .38$ & 0.16 \\
\hline $\mathrm{N}$ iños <-1Z peso/edad (\%) & $102(44.9)$ & $91(40)$ & $11(4.8)$ & 0.29 \\
\hline Hemoglobina (g/dl) & $11.03 \pm 1.33$ & $11.86 \pm 1.37$ & $.64 \pm 1.93$ & $<0.001$ \\
\hline $\bar{N}$ iños anémicos (\%) & $36(15.8)$ & $18(7.9)$ & $18(7.9)$ & 0.003 \\
\hline Hierro sérico ( $\mu \mathrm{g} / \mathrm{dl})$ & $108.1 \pm 44$ & $114.8 \pm 31.3$ & $6.42 \pm 48.9$ & 0.06 \\
\hline$\overline{N \text { iños con deficiencia de hierro(\%) }}$ & $58(25.5)$ & $18(7.9)$ & $40(17.6)$ & $<0.001$ \\
\hline Saturación de hierro, \% & $31.5 \pm 15.3$ & $31 \pm 9.3$ & $-.89 \pm 17.04$ & 0.65 \\
\hline Captación total de hierro $(\mu \mathrm{g} / \mathrm{dl})$ & $351 \pm 105$ & $380.6 \pm 58$ & $32.25 \pm 111$ & 0.0001 \\
\hline Ácido fólico, ng/ml & $12.8 \pm 7.7$ & $24.8 \pm 10.5$ & $7.75 \pm 7.05$ & 0.0001 \\
\hline$\overline{\text { Vitamina } B_{12}, p g / m l}$ & $649 \pm 494.2$ & $1053 \pm 854$ & $413 \pm 839$ & 0.001 \\
\hline
\end{tabular}

$* D$ atos notificados como $\bar{X} \pm D E$, a menos que se especifique otra medición 


\section{Cuadro V \\ Comparación de la prevalencia relativa DE DESNUTRICIÓN A LA ADMISIÓN \\ Y AL FINALIZAR LA SUPLEMENTACIÓN CON LECHE. Ciudad de México, 1998}

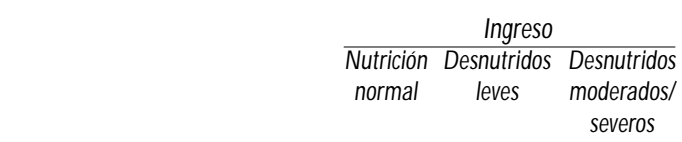

\begin{tabular}{|c|c|c|c|c|}
\hline Nutrición normal & 75 & 15 & 2 & 192 \\
\hline D esnutridos leves & 7 & 18 & 5 & 30 \\
\hline D Desnutridos moderados/severos & 0 & 3 & 2 & 5 \\
\hline & 82 & 36 & 9 & 227 \\
\hline
\end{tabular}

$\chi^{2}=102.54$

$p<0.0001$

\section{Discusión}

El presente estudio da cuenta del impacto nutricional, la aceptación y el apego a la suplementación con micronutrimentos mediante el consumo de una leche entera ultrapasteurizada, administrada en un volumen de $500 \mathrm{ml}$ al día, durante 90 días, a una población de niños menores de cinco años en la ciudad de México.

Este estudio es el primero que se realiza en México entre población infantil abierta, para valorar el impacto de la suplementación con vitaminas y minerales en una leche entera ultrapasteurizada que se encuentra lista para su consumo y a la venta en el mercado nacional.

De acuerdo con la base de datos global de nutrición de la OMS (1987), que cubre a $87 \%$ de la población de niños menores de cinco años en los países en desarrollo, se estima que más de un tercio de los niños se encuentran con un estado nutricional deficiente; Asia y África son los continentes con mayor prevalencia. En conjunto, 80\% de los niños desnutridos del mundo viven en Asia, África y América Latina, y en esta última región se encuentra el 5\%. Se desconoce la prevalencia de deficiencias específicas de micronutrimentos en el mundo, ${ }^{26}$ aunque en México se está realizando la segunda Encuesta Nacional de Nutrición, que incluye la evaluación del estado de micronutrimentos y que en los siguientes meses nos dará información importante.

En el presente estudio la población infantil tuvo al ingreso una condición nutricia aceptable, ya que se encontró una prevalencia de desnutrición moderada a severa ( $<-2 \mathrm{Z}$ peso / talla) de 3.9\% y una media del valor $\mathrm{Z}$ peso / talla de -0.35 . Según las definiciones propuestas por la $\mathrm{OMS}^{27-30}$ se establece que el estado de nutrición de una población es aceptable cuando existe una prevalencia de desnutrición moderada a severa $<5 \%$ y una media de Z peso / talla $>-0.4$. El informe de la OMS para México, en 1988, informó una prevalencia de 5.5\% del indicador $>-2 \mathrm{Z}$ peso / talla; esta cifra nacional es mayor a la encontrada en esta población estudiada, lo que pudiera estar relacionado con las características de este lugar suburbano de la ciudad de México y con la diferencia que existe de 10 años entre el informe publicado por la OMS y el presente trabajo. Además, la población estudiada no es representativa de todo nuestro país.

De acuerdo con este trabajo, la prevalencia de desmedro de los sujetos de estudio a su ingreso puede catalogarse como baja, según los criterios de la OMS ya referidos.

El apego y la aceptación de leche entera fortificada fueron adecuados en la población, sin que se pudieran observar diferencias entre el grupo con desnutrición y el grupo con estado nutricio normal; ello demuestra que este tipo de leche es aceptable para la población infantil de 8 a 36 meses y que no es necesario adicionarle saborizantes ni colorantes para su consumo, independientemente del estado nutricional de los menores.

Otro estudio realizado en México por Rosado y colaboradores ${ }^{31}$ evaluó la aceptación y el apego al consumo de una leche fortificada con vitaminas y micronutrimentos, y adicionadas con diversos saborizantes; de este modo se determinó qué sabor producía más apego y aceptación en 14 días de consumo. Se encontró una aceptación y un apego similar entre las mujeres embarazadas y en periodo de lactancia, tanto con los productos saborizados como con aquellos a los que no se les añadió sabor; sin embargo, cuando se evaluó en los niños, se presentó un consumo superior cuando se adicionaban saborizantes. Los resultados notificados de apego y consumo de leche con sabor entre los niños del estudio de Rosado y colaboradores son similares a los del presente trabajo.

Las deficiencias de vitaminas y minerales resultaron similares entre los niños mal nutridos y aquellos con peso/talla adecuados. Esto es consistente con observaciones previas sobre la prevalencia de desnutrición por micronutrimentos, a partir de los cuales se ha encontrado población infantil con índices antropométricos de desnutrición pero sin deficiencias de micronutrimentos, así como población infantil con índices de crecimiento normal pero con deficiencia selectiva de micronutrimentos. La prevalencia de anemia también fue similar entre los grupos con diferente estado nutricio.

Posterior a la suplementación con la leche fortificada, la Z de peso / talla mostró una diferencia significativa en comparación con la que se observó al ingreso. El indicador Z talla/edad presentó una disminución, aunque no significativa, probablemente debido a que la desnutrición crónica evidenciada por la baja talla/edad 
requiere de mayor tiempo para su erradicación. En este estudio el tiempo de suplementación fue breve.

Al término de la investigación se encontró un incremento significativo en la concentración de hemoglobina y de hierro plasmático, en comparación con las determinaciones iniciales; asimismo, se observó una disminución importante en el porcentaje de niños anémicos y una reducción significativa en la prevalencia de deficiencia de hierro. La concentración de ácido fólico y de vitamina $B_{12}$ también presentó un incremento significativo al finalizar el estudio.

El porcentaje de niños con un indicador peso/ talla $<-1 Z$ disminuyó de 19.8 a 15.4\%, lo cual significa que la población mejoró su estado nutricio. El número de desnutridos severos también disminuyó de nueve niños al inicio, a cinco niños al finalizar, lo cual señala la misma tendencia. Probablemente se requiera de mayor tiempo de suplementación para disminuir aún más el número de niños con presencia de desnutrición severa, hasta erradicar este padecimiento. Otra explicación es, indudablemente, el tamaño de la muestra de niños con déficit de peso para la talla, pues no permite generar conclusiones definitivas para este tipo de población. Es necesario realizar otro estudio que evalúe el impacto de esta leche fortificada en una población infantil con índice peso / talla $<-2 Z$.

Una limitante de este estudio es que no se estableció una comparación con un grupo suplementado con leche sin micronutrientes, y ello no se hizo porque nuestra población de niños era heterogénea y por consideraciones éticas, ya que el beneficio potencial de la suplementación con vitaminas y minerales era muy probable.

En este trabajo se concluye que la suplementación con vitaminas y minerales, usando la leche entera ultrapasteurizada como vehículo, es ampliamente aceptada entre los niños menores de cinco años de nuestro país y que, además, mejora el estado nutricio, así como la concentración de hemoglobina, vitamina $\mathrm{B}_{12} \mathrm{y}$ folatos; asimismo, reduce la prevalencia de anemia y de deficiencia de hierro.

Se sugiere la realización de este tipo de estudios de suplementación en población menor de cinco años de edad, con estratos de índice peso / talla <-2Z más representativos y por periodos de suplementación más prolongados; incluso, se recomienda evaluar su impacto en el crecimiento longitudinal y la talla para la edad.

\section{Referencias}

1. Beaton $\mathrm{GH}$, Martorell R, Aronson KJ, Edmonston $A B$, McC abe $G$, Ross $A C$ et al. Effectiveness of vitamin $A$ supplementation in the control of young child mortality in developing countries. Ginebra: ACC/SCN (N utrition Policy Discussion, núm. 13), 1993.

2. Fawzi W W, Herrera MG, W illet W C, N estel P,Amin EL, Lipsitz SL et al. Dietary vitamin $A$ intake and the risk of mortality among children. Am J Clin N utr 1994;59:401-408.

3. Ross DA, Kirkwood BR, Binka FN ,A rthur P, D ollimer N, Morris SS et al. Child morbidity and mortality following vitamin $A$ supplementation in Ghana.Time since dosing, number of doses and time of year. A $m$ J Public Health 1995;85:1246-1251.

4. Rosado JL, López P, Muñoz E, Martínez H ,Allen LH. Zinc supplementation reduced morbidity, but neither zinc nor iron supplementation affected growth or body composition of Mexican preschoolers. Am J Clin Nutr 1997; 65(1):13-19.

5. Rivera J, Rosado JL, Brown K, Flores M, González-C ossio T, Rivera M. Effect of multiple micronutriment supplementation on the growth of young rural Mexican children. FASEB J 1996;A 290:23-29.

6. Manson D, Franco D, Arbeter $H$, Vélez $H$, Vitale JJ. Serum levels of immunoglobulins, cell-mediated immunity, and phagocytosis in proteincalorie malnutrition. Am J C lin N utr 1977;27:625-628.

7. Schlesinger L, Stekel A. Impaired cellular immunity in marasmic infants. Am J Clin N utr 1997;27:615-620.

8. Black RE, Lanata CF, Lazo F. D elayed cutaneous hypersensitivity: Epidemiologic factors affecting and usefulness in predicting diarrheal incidence in young Peruvian children. Pediatr Infect D is J 1989;8:210-215. 9.G reen F, H eyworth B. Immunoglobulin-containing cells in jejunal mucosa of children with protein-energy malnutrition and gastroenteritis.Arch $D$ is Child 1980;55:380-383.

10. Rosado JL, Bourges H, Saint Martin B. Deficiencia de vitaminas y minerales en México: una revisión crítica de información.I. D eficiencia de minerales. Salud Publica Mex 1995;37:130-139.

11. Rosado JL, Bourges H, Saint-Martin B. Deficiencia de vitaminas y minerales en México. Una revisión crítica del estado de la información. II. D efiencia de vitaminas. Salud Publica Mex 1995;37:452-461.

12. Vega-Franco L, Meza-Camacho C, Meijenrik J, A legretila $C$. Concentración de vitamina $E$ en niños con desnutrición proteicoenergética. Bol Med Hosp Infant Mex 1989;46:607-610.

13. Vega-Franco L, De León S, Meza C. Variación estacional de la concentración de ácido ascórbico en niños de la Ciudad de México. Bol Med Hosp Infant Mex 1978;35:1141-1145.

14. Madrigal $H$, Avila A, Castro JM, Morales M, Gómez G, López $R$ et al. Encuesta $\mathrm{N}$ acional de Alimentación en el Medio Rural, 1989. México, D.F.: División de N utrición de Comunidad-IN N SZ (Publicación L-89), 1990.

15. Rappeti MC, D onato $H$, de Galvagni A, Lubovitsky M, Lanzilotta M, Trepacka $E$ et al. Correction of iron deficiency with an iron fortified fluid whole cow's milk in children: Results of a pilot study. J Pediatr Hematol Oncol 1997;19(3):192-196.

16. Chandra RK, SorayaAK. Impaired immunocompetence associated with iron deficiency. J Pediatr 1975;86:899-902.

17.Gardner GW, Edgerton VR, Senewiratne B, Barnard RJ, Yoshinobu 0. Physical work capacity and metabolic stress in subjects with iron deficiency anemia.Am J Clin N utr 1977;30:910-917.

18.W alter T,Arredon S, Stekel AM. Effect of iron therapy on phago cytosis and bacterial activity in neutrophils of iron deficient infants. Am J Clin N utr 1986;44:877-882.

19. Sheshadri S, G opaldasT. Impact of iron supplementation on cognoscitive functions in preschool and school age children:The Indian experience.Am J Clin Nutr 1989;50:675-686.

20. Informe de avance sobre la propuesta en práctica de la Declaración Mundial y Plan de Acción de la Conferencia Internacional de Nutrición. Roma/Ginebra: FAO/O MS, 1996.

21. O rganización Panamericana de la Salud/O rganización Mundial de la Salud. Plan Regional de Alimentación y N utrición.W ashington, D.C :: O PS/ O MS, 1997. 
22. Progresa. Programa de Educación, Salud yA limentación. Poder Ejecutivo Federal. 1995-2000. México, D.F.: , : : 38-64.

23. Manual del 0 perador Sysmex F-800. Barcelona/Córcega: Técnicas Médicas MAB, 1989.

24. Loria A. Modificación del método de Beale et al. J Clin Pathol 1962:15:156-163.

25. G uidelines for evaluating a B12 (cobalamin) assay. $N$ ational Committee for Clinical Laboratory Standard, 1980.

26. D e 0 nis M, Monteiro $C$, A kré J, C lugston $G$. The worldwide magnitude of protein-energy malnutrition:A n overview from theW HO Global D atabase on C hild G rowth. Bull W orld Health 0 rgan 1993; 71(6):703-712.

27. Gorstein J, Sullivan K,Yip R, De 0 nis M, Trowbridge F, Fajans $P$ et al. Uses in the assessment of nutritional status using anthropometry. Bull W orld Health 0 rgan 1994;72:272-283.
28. W aterlow JC, Buzina R, Kelleer W, Lane JM, N ichaman MZ,Tanner JM. The presentation and the use of height and weight data for comparing the nutritional status of groups of children under the age of 10 years. Bull W orld Health 0 rgan 1977;55:489-498.

29. W orld Health O rganization. Measuring change in nutritional status. Ginebra:W HO, 1983.

30.W orld Health 0 rganization.W orking Group. Use and the interpretation of anthropometric indicators of nutritional status. Bull W orld $\mathrm{H}$ ealth $\mathrm{O}$ rgan 1986;64:929-941.

31. Rosado JL, Rivera J, López G, Solano L, Rodríguez G , C asanueva E et al. Desarrollo y evaluación de suplementos alimenticios para el Programa de Educación, Salud y Alimentación. Salud Publica Mex 1999; 41(3):153-162. 